\title{
Monitoring System for the Gold Target by Radiation Detectors in Hadron Experimental Facility at J-PARC
}

\author{
Ryotaro Muto ${ }^{1, *}$, Keizo Agari ${ }^{1}$, Kazuya Aoki ${ }^{1}$, Kotaro Bessho ${ }^{1}$, Masayuki Hagiwara ${ }^{1}$, Erina Hirose $^{1}$, Masaharu Ieiri $^{1}$, \\ Ruri Iwasaki ${ }^{1}$, Yohji Katoh $^{1}$, Jun-ichi Kitagawa ${ }^{1}$, Michifumi Minakawa ${ }^{1}$, Yuhei Morino ${ }^{1}$, Kiwamu Saito ${ }^{1}$, Yoshinori Sato ${ }^{1}$, \\ Shin'ya Sawada ${ }^{1}$, Yoshihisa Shirakabe ${ }^{1}$, Yoshihiro Suzuki ${ }^{1}$, Hitoshi Takahashi ${ }^{1}$, Kazuhiro Tanaka ${ }^{1}$, Akihisa Toyoda $^{1}$, \\ Hiroaki Watanabe ${ }^{1}$, and Yutaka Yamanoi $^{1}$
}

${ }^{1}$ High Energy Accelerator Research Organization, 1-1 Oho, Tsukuba, Ibaraki 3050801, JAPAN

\begin{abstract}
At the Hadron Experimental Facility in J-PARC, we inject a $30-\mathrm{GeV}$ proton beam into a gold target to produce secondary particle beams required for various particle and nuclear physics experiments. The gold target is placed in a hermetic chamber, and helium gas is circulated in the chamber to monitor the soundness of the target. The radioactivity in helium gas is continuously monitored by gamma-ray detectors such as a germanium detector and a $\mathrm{NaI}(\mathrm{Tl})$ detector. Beam operations with those target-monitoring systems were successfully performed from April to June and October to December 2015, and from May to June 2016. In this paper, the details of the helium gas circulation system and gamma-ray detectors and the analysis results of the obtained gamma-ray spectra are reported.
\end{abstract}

\section{Introduction}

The Hadron Experimental Facility (HEF) is one of the experimental facilities in Japan Proton Accelerator Research Complex (J-PARC) [1] located in Tokai, Ibaraki, Japan. In the HEF, we inject the proton beam into a gold target to produce secondary particles such as kaons and pions and use them for various particle and nuclear experiments [2]. Figure 1 shows the accelerator configuration of the J-PARC and Figure 2 shows the schematic view of the HEF.

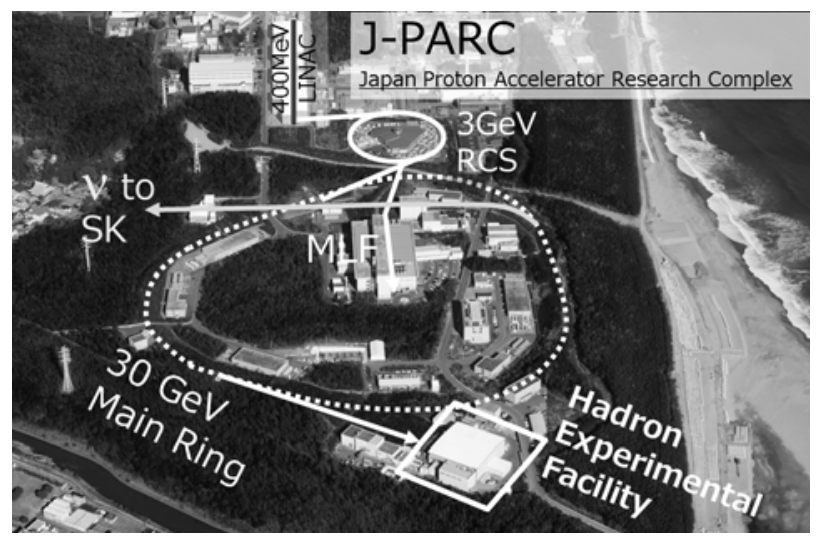

Fig. 1. Accelerator configuration of the J-PARC. Protons are accelerated up to $30 \mathrm{GeV}$ through the $400-\mathrm{MeV}$ linac, $3-\mathrm{GeV}$ Rapid Cycle Synchrotron, and 30-GeV Main Ring and slowly extracted to the Hadron Experimental Facility (HEF).

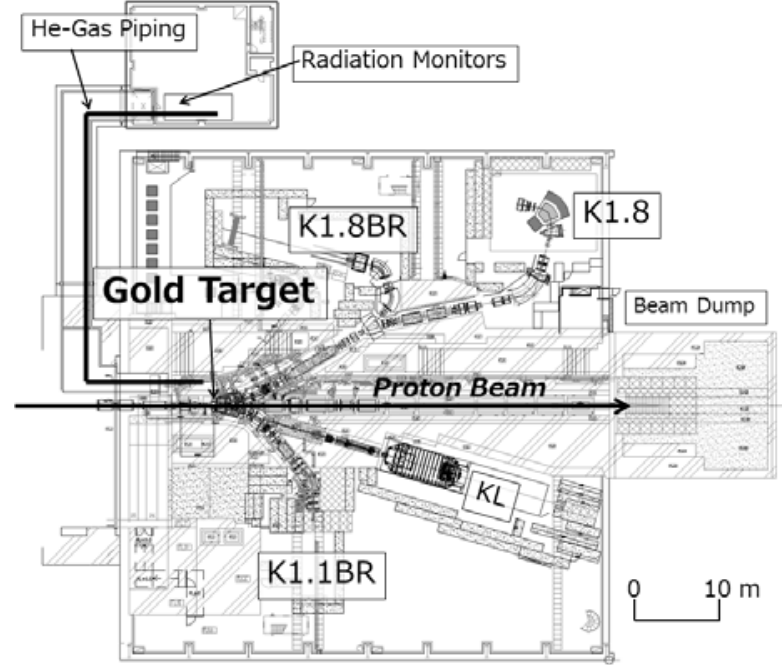

Fig. 2. Schematic view of the HEF. The secondary particles produced at the gold target are delivered to each experimental area. The locations of He-gas piping and radiation monitors for the monitoring system are also shown.

The proton beam accelerated up to $30 \mathrm{GeV}$ is slowly extracted from the Main Ring for 2 seconds within 6 or 5.52 seconds-accelerator cycles and injected into the gold target [3]. The gold target is bonded onto a watercooled copper base by the heat isostatic pressing (HIP) method. Figure 3 shows a photograph and a schematic cross section of the gold target.

* Corresponding author: ryotaro.muto@kek.jp 


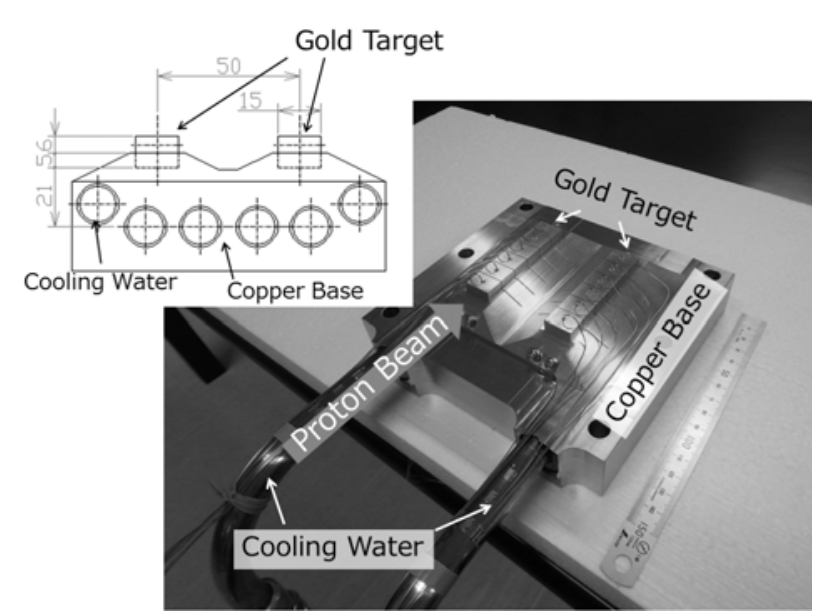

Fig. 3. Photograph and schematic cross section of the gold target. Two gold targets are mounted on a water-cooled copper base: one is for the beam operation and the other is a spare.

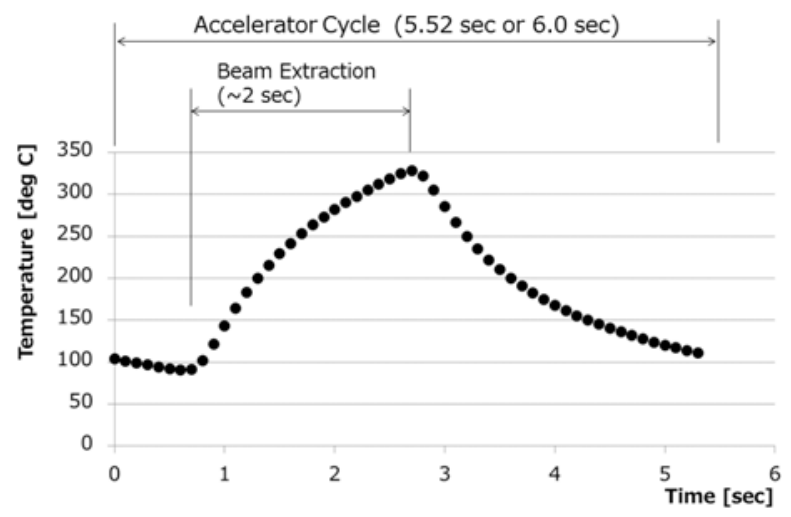

Fig. 4. Typical target temperature variations during an accelerator cycle. The temperature is measured at the top of the target by a thermocouple. The target temperature rises during the beam extraction, which lasts approximately 2 seconds, and then decreases. The maximum temperature is approximately $320^{\circ} \mathrm{C}$ with the $42-\mathrm{kW}$ beam.

The temperature of the target is measured by thermocouples, and it reaches a maximum of $350{ }^{\circ} \mathrm{C}$ with the normal $50-\mathrm{kW}$ beam. Figure 4 shows the typical temperature variation of the gold target measured with a $42-\mathrm{kW}$ proton beam. The target temperature depends not only on the beam power but also on the position, profile and instantaneous rate of the extracted beam; thus, these beam properties are continuously monitored. When a large deviation from the normal operation is detected, the beam extraction stops within several milliseconds. In addition to these measures to protect the target, a hermetic chamber for the gold target and a helium gas circulation system with gamma ray detectors are installed. In case of target failure, the temperature of the target rises abnormally and the accumulated radioactive materials are released from the damaged target [4]. The hermetic chamber prevents the radioactive materials from spreading. To detect such events the chamber is filled with circulating helium gas and the rate and energy spectrum of the gamma rays from radionuclides in helium gas are continuously monitored by a germanium detector and a $\mathrm{NaI}(\mathrm{Tl})$ detector.

\section{Helium gas circulation system}

Figure 5 shows a schematic view of the helium gas circulation system.

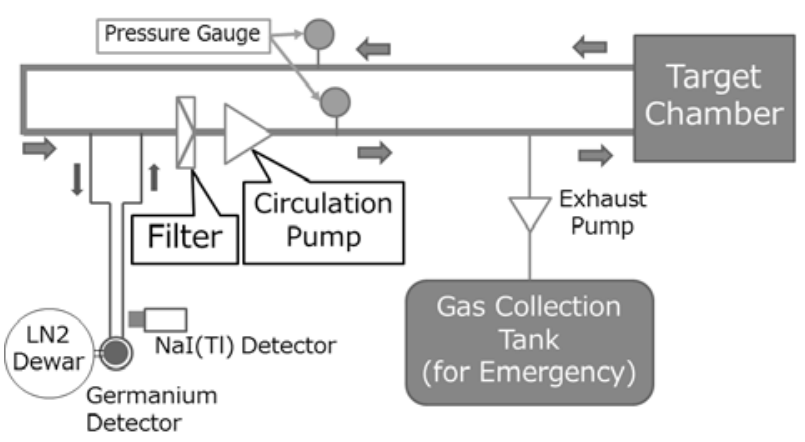

Fig. 5. Schematic view of the helium gas circulation system.

The helium gas circulation system is placed in the machine building located next to the Hadron Experimental Hall. The target chamber and circulation system are connected using gas pipes, which are 168-m long and have a 11/4-inch nominal diameter for the most part. The total volume of helium gas in the target chamber and gas pipes is $0.35 \mathrm{~m}^{3}$ with a pressure of $80 \mathrm{kPa}$. The gas is circulated by a metal bellows pump and its flow rate is $3.6 \mathrm{~m}^{3} /$ hour. A filter unit composed of a HEPA filter and a charcoal filter is mounted to reduce contaminations in the gas. The status of the circulation system is monitored by pressure gauges, flowmeters, a dew-point meter and a helium purity monitor. Approximately $10 \%$ of the gas branches off to be monitored by gamma-ray detectors. The flow rate in the branch line is $0.35 \mathrm{~m}^{3} /$ hour. The radioactive materials generated in the target chamber are estimated to reach the detectors in approximately $90 \mathrm{~s}$. In case the gamma-ray detectors observe high-rate gamma rays in helium gas, the gas is collected in a gas collection tank to localize the radioactive materials. The $99 \%$ gas can be collected less than 10 minutes in the tank.

The hermetic target chamber has two beam windows made of titanium alloy (Ti-6Al-4V). The thicknesses of the beam-entry and beam-exit windows are 2 and $4 \mathrm{~mm}$, respectively. Further details of the target chamber could be found in [3].

\section{Germanium detector for monitoring radioactivity in the circulating helium gas}

The spectra and rate of the gamma rays from helium gas are acquired and monitored by a high-purity germanium detector system made by Mirion Technologies (Canberra), Inc. [5]. We referred to the concepts of the previous monitoring system built for the neutron production target at JSNS [6] for developing the system. The energy resolution of the germanium detector is less than $1.8 \mathrm{keV}$ in FWHM at $1332 \mathrm{keV}$ and the energy range covered is from $40-4000 \mathrm{keV}$. Figure 6 shows photographs of the germanium detector system. The dimensions of the detector are shown in Figure 7. The helium gas pipe winds twice around the detector. The 
total efficiency for the detection of the gamma rays in the gas system is estimated to be $8 \times 10^{-7}$ at $1332 \mathrm{keV}$. The detector and gas pipe are covered by $100-\mathrm{mm}$-thick lead shields to suppress backgrounds, and 3-mm-thick copper plates are placed between the lead shields and the detector to reduce the effect of X-rays from lead shields. The thickness of the copper plates was determined according to [7]. Figure 8 shows the gamma-ray spectra with and without the copper plates. The copper plates effectively reduce $\mathrm{X}$-rays in the energy range below $100 \mathrm{keV}$. The detector is cooled by the liquid nitrogen stored in a dewar. The dewar has a pulse-tube cryocooler and there is no need to add liquid nitrogen during a beam operation which typically lasts several months.

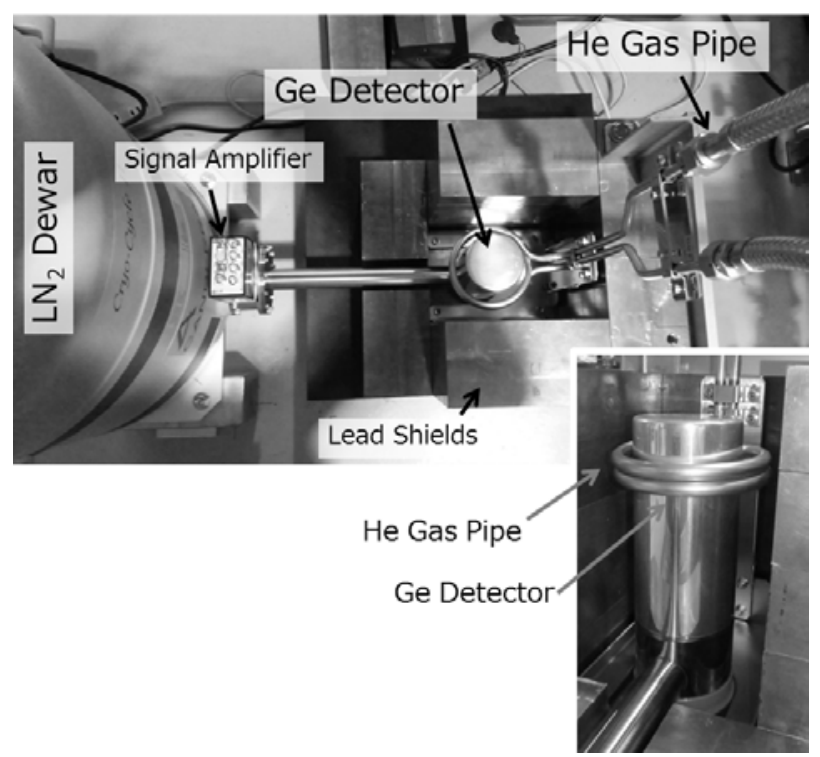

Fig. 6. Photographs of the germanium detector system without copper plates and a part of lead shields. The lower right photograph shows the zoomed-in view of the germanium detector and helium gas pipe winding around the detector.

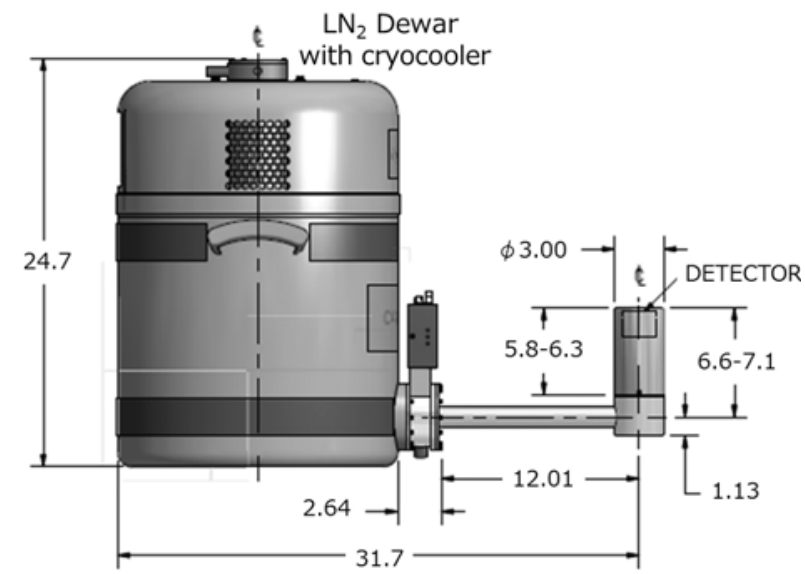

Fig. 7. Dimensions of the germanium detector system in inches.

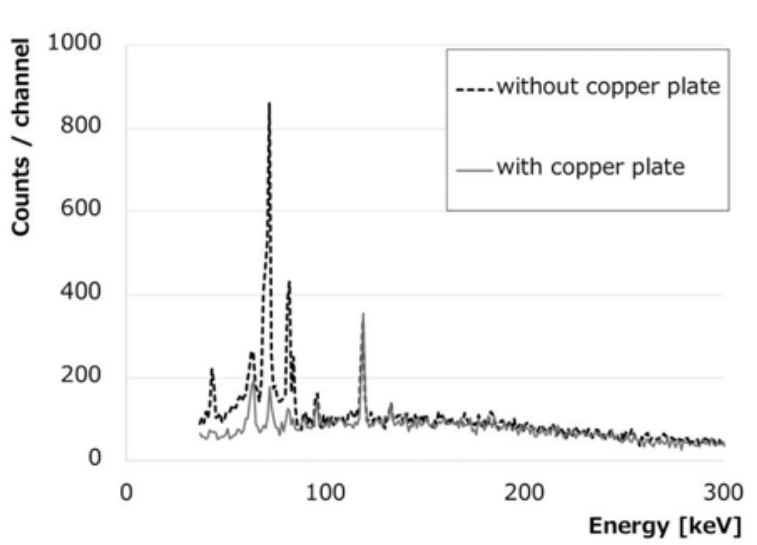

Fig. 8. Gamma-ray spectra with and without the copper plates between the lead shields and the germanium detector.

\subsection{Data processing of the germanium detector}

Figure 9 shows the schematic configuration of the data processing system of the germanium detector. The pulse height of the signals from the germanium detector are analyzed and digitized by a multichannel analyzer (MCA), and collected by a desktop computer located near the germanium detector. The desktop computer has a contact output and produces a beam abort signal when the following parameters exceed the predetermined thresholds:

- Total counting rate in the energy range from $40 \mathrm{keV}$ to $4 \mathrm{MeV}$

- One hour accumulations of the gamma-ray counts in ten energy ranges of interest for focused monitoring of the nuclides expected to be produced in the target and the beam windows

- The dead time of the measurement (to detect the saturation of the detector due to a large number of gamma rays)

- Network response time between the desktop computer and the database server

The beam operation stops within several milliseconds after the beam abort signal is detected.

The energy spectra accumulated in an hour and the total counting rate averaged in a minute are transferred via the network to a database server (Microsoft SQL server) located in a control room. The acquired data are stored in a RAID 5 disk array and backed up in a network attached storage (NAS) once a day.

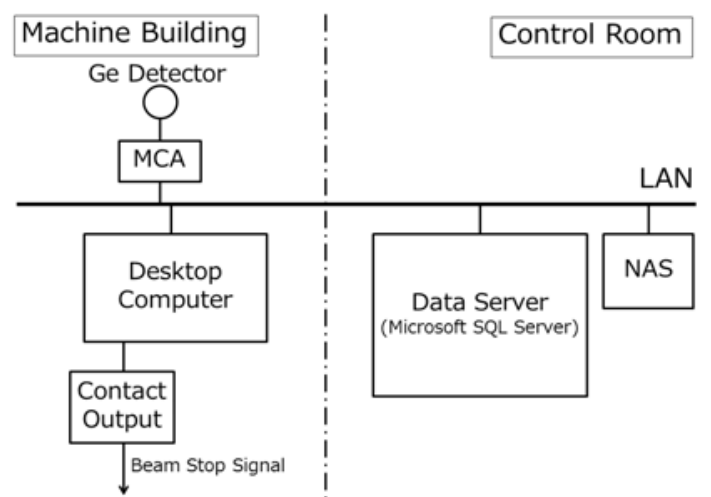

Fig. 9. Schematic diagram of the system used to process the data from the germanium detector. 


\section{$4 \mathrm{Nal}(\mathrm{TI})$ detector for monitoring the rate of gamma rays from the circulating helium gas}

The rate of the gamma rays is also monitored by a $\mathrm{NaI}(\mathrm{Tl})$ detector, which has a higher rate tolerance, made by Mirion Technologies (Canberra), Inc. [5]. It is mounted just above the lead shields of the germanium detector. Figure 10 shows a photograph of the $\mathrm{NaI}(\mathrm{Tl})$ detector. The detector is composed of a $2 \times 2$ inch $\mathrm{NaI}(\mathrm{Tl})$ crystal and a photomultiplier and covered by 50 mm-thick lead shields. The detector is placed at a distance of $10 \mathrm{~mm}$ from the helium gas pipe and the total detection efficiency is estimated as $6 \times 10^{-7}$ at $1332 \mathrm{keV}$. The counting rate of the gamma rays whose energies are larger than $250 \mathrm{keV}$ is converted into a voltage by an ORTEC Model 661 Ratemeter [8]. If the Ratemeter output exceeds the predetermined threshold level, the beam extraction is immediately aborted.

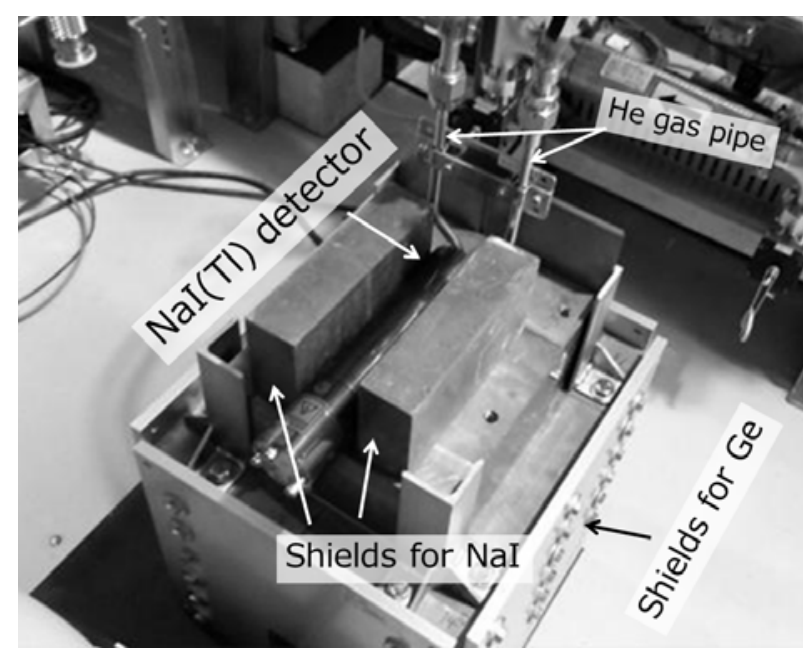

Fig. 10. Photograph of the $\mathrm{NaI}(\mathrm{Tl})$ detector without lead shields at the top and in front of the detector.

\section{Beam operations with the gamma-ray detectors and helium gas circulation system}

The installation of the system described above was completed by March 2015 and beam operations were performed from April to June and October to December 2015, and from May to June 2016. The beam power in the operations was $3 \mathrm{~kW}$ initially and gradually increased up to $42 \mathrm{~kW}$. The data from germanium and $\mathrm{NaI}(\mathrm{Tl})$ detectors were successfully acquired through the beam operations.

\subsection{Identified radioactive nuclides}

Figure 11 shows an example of the gamma-ray spectrum in the $42-\mathrm{kW}$ normal beam operation accumulated in an hour. From the spectrum, $\mathrm{Hg}-192$, which is presumed to be produced in the gold target and transported to the detector in the gaseous phase, and its daughter nuclide $\mathrm{Au}-192$ were identified by the peak positions and half- lives. C-10, O-14, O-19, Ne-23, Ne-24, Na-24, Ar-41, Sc-44m, I-119, I-121, I-123, Xe-123, Xe-125, Hg-193m, and $\mathrm{Hg}-195 \mathrm{~m}$ were also identified.

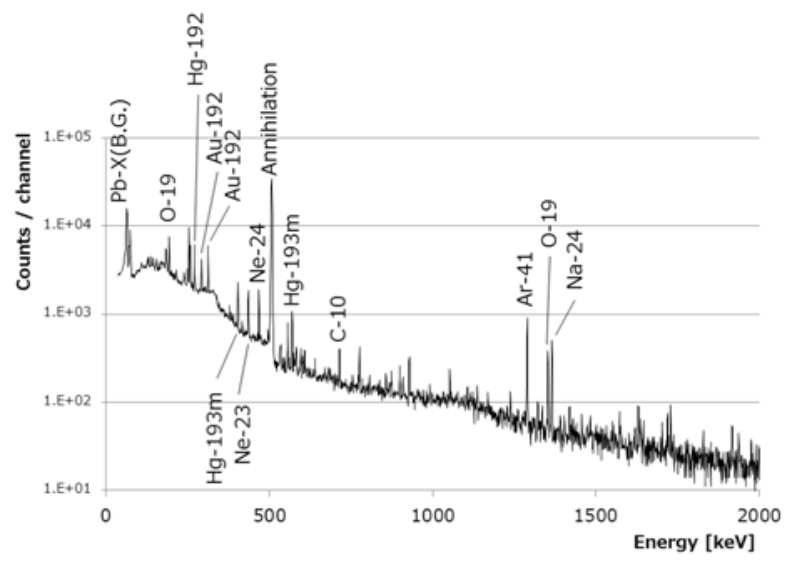

Fig. 11. Example of the gamma-ray spectrum obtained by the germanium detector. The spectrum was accumulated in an hour with the $42-\mathrm{kW}$ beam. The major radionuclides identified by the energy and half-lives are indicated.

Figure 12 shows the trend graphs of the counting rates of the germanium detector corresponding to the radioactive nuclides identified with the $42-\mathrm{kW}$ beam. The amount of each radioactive nuclide is stably monitored during the beam operation.

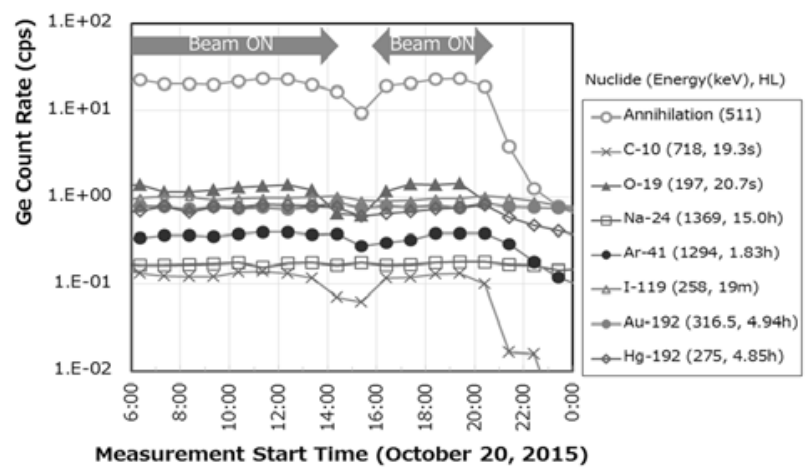

Fig. 12. Counting rates of the germanium detector corresponding to the identified radioactive nuclides as a function of measurement start time. Each counting rate is derived from the gamma ray spectrum accumulated in an hour.

\subsection{Response time of the germanium detector for the radioactive materials produced in the target chamber}

The target chamber and gamma-ray detectors are connected using 168-m long helium gas pipes. Thus it takes non-negligible time for the radioactive nuclides produced in the target chamber to reach the gamma-ray detectors. We measured this response time at the start of the beam operation. Figure 13 shows the results. The count rate of the germanium detector increases approximately 50 seconds after the start of the beam operation in both the first and second measurements. With this result, we establish a regulation for the beam operation: when an abnormal beam condition is detected, we watch the counting rate and the spectrum of the 
germanium detector carefully for $5 \mathrm{~min}$ to determine the soundness of the gold target.

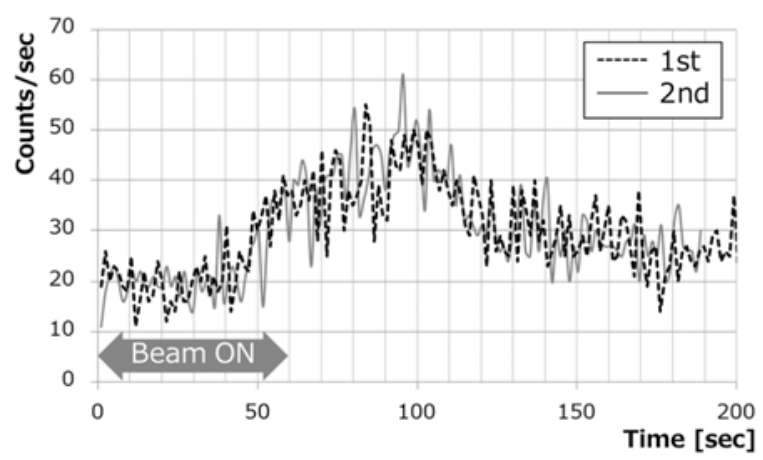

Fig. 13. Results of the response time measurements for the germanium detector. The beam operation started at time 0 and lasted for 60 seconds in this measurement.

\subsection{Dependence of the amount of radioactive nuclides on beam power and target temperature}

The radioactive nuclides detected by the gamma-ray detectors are mainly produced in the gold target and the titanium-alloy beam windows of the target chamber, and the amounts of radioactive nuclides depend on the production rates and release rates of the nuclides in the bombarded materials. Thus, we investigated the dependences of the count rates for the identified radioactive nuclides on the beam power and the target temperature. Figure 14 shows the measured maximum target temperature as a function of the beam power, which increases approximately linearly with the beam power. We performed two beam operations with the same beam power of $42 \mathrm{~kW}$ in the winter of 2015 and spring of 2016, and the maximum target temperature in the latter beam period $(302 \mathrm{~K})$ was higher than that of former $(277 \mathrm{~K})$ because of the shorter beam extraction duration and the beam position difference.

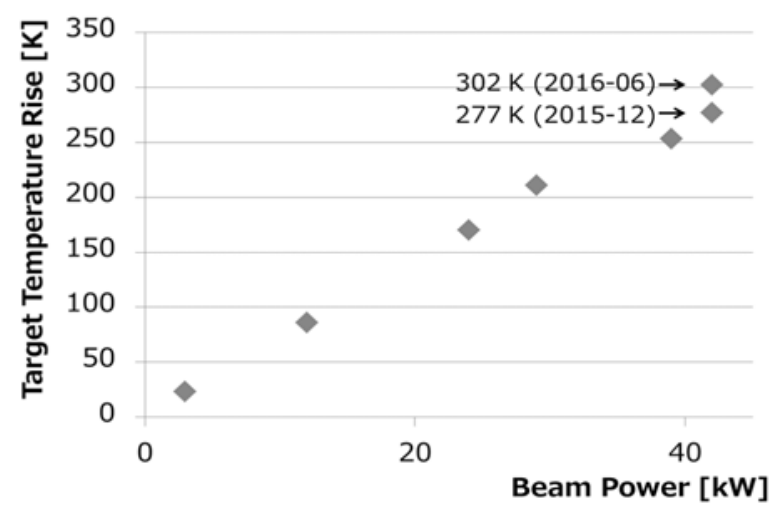

Fig. 14. Maximum target temperature rise as a function of the beam power.

Figure 15 shows the dependences of the counting rates for the identified radionuclides measured by the germanium detector. We used the data from the winter run of 2015 (target temperature rise was $277 \mathrm{~K}$ ) for the $42 \mathrm{~kW}$ beam power. Lighter nuclides such as O-19, Ne-
23, Na-24, and Ar-41 have approximately linear dependence on the target temperature. On the other hand, heavier nuclides such as Au-192 (daughter of Hg-192), $\mathrm{Hg}-192, \mathrm{Hg}-193 \mathrm{~m}$, and Hg-195m are observed only with the beam powers higher than $29 \mathrm{~kW}$. This indicates that there are factors other than the beam power to determine the amount of detected radionuclides.

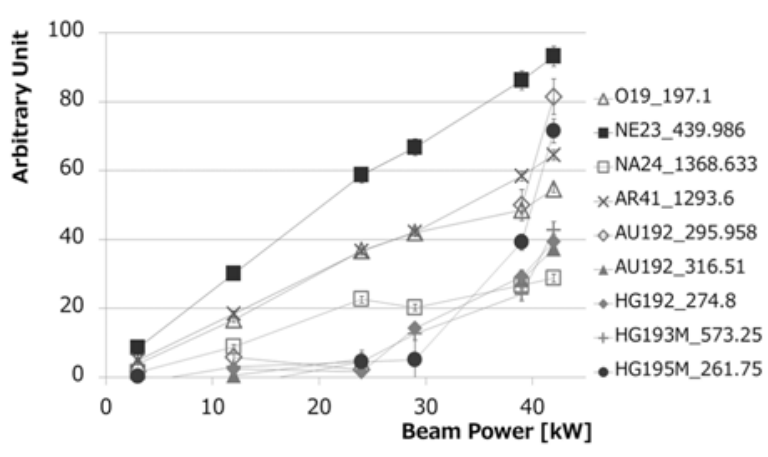

Fig. 15. Beam power dependence of the amount of the radionuclides identified by the germanium detector.

Thus, we investigated the target temperature dependence of the counting rates of the identified radionuclides using two beam operations with the same beam power of $42 \mathrm{~kW}$ and different target temperature rises $(277 \mathrm{~K}$ and $302 \mathrm{~K})$. Figure 16 shows the ratios of the counting rates of the radionuclides in $302 \mathrm{~K}$ data to $277 \mathrm{~K}$ data. In the ratios for nuclides lighter than Ar-41, the dependence on the target temperature is not clearly seen, which implies that those lighter nuclides were mainly produced in the titanium-alloy beam windows. By contrast, nuclides heavier than I-121, which are considered to be produced in the gold target, have ratios ranging from 2 to 4 . The radionuclides produced in the gold target diffuse to its surface and are released into the circulating helium gas, thus the temperature dependence of the counting rates of radionuclides is possibly explained by the combination of the temperature dependence of the diffusion and release processes. First, the diffusion in a solid state material is governed by Fick's first law, and the temperature dependence of the diffusion coefficient $D$, which is an essential parameter characterizing the diffusion process, is described by the Arrhenius equation $D(T)=D_{0} \exp \left(-E_{a} / k T\right)$, where $E_{a}$ is the activation energy for diffusion [9]. Second, the release rate also depends on the temperature. For example, the vapor pressure of mercury at $600 \mathrm{~K}$ is approximately twice that at $570 \mathrm{~K}$ [10]. Those factors certainly depend on the nuclide, but the measurement accuracy is insufficient for the discussion on nuclide dependence. In future beam operations, we will collect data with different beam powers and temperatures and perform further studies. 


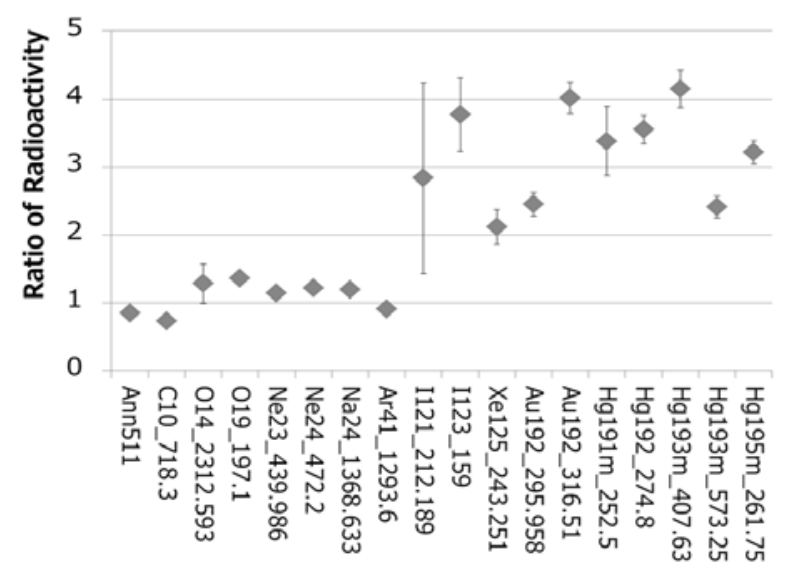

Fig. 16. Ratios of the counting rates of the identified radionuclides in $302-\mathrm{K}$ data to those in $277-\mathrm{K}$ data.

\section{Summary}

We developed a target monitoring system composed of a hermetic target chamber, helium gas circulation system, and gamma-ray detectors and successfully operated the system for beam operations. Several radioactive nuclides produced by the beam were identified, and it was shown that the amounts of radioactive nuclides depend on the target temperature as well as beam power. The next beam operation is scheduled for March 2017 and further studies will be done.

\section{References}

1. Joint Project Team of JAERI and KEK, KEK Report 99-4 (1999) and JAERI-Tech 99-056 (1999)

2. K. Agari et al., Prog. Theor. Exp. Phys., 02B009 (2012)

3. H. Takahashi et al., Journal of Radioanalytical and Nuclear Chemistry 305-3 (2015) 803-809

4. M. Hagiwara et al., JPS Conf. Proc., 8 (2015) pp. 051007-1 - 051007-6

5. http://www.canberra.com/

6. Y. Kasugai, K. Otsu and T. Kai, Proceedings of the ICANS-XIX, 19th meeting on Collaboration of Advance Neutron Source (2010)

7. K. Komura, RADIOISOTOPES, 55 (2006) 233-243 (in Japanese)

8. http://www.ortec-online.com/download/661.pdf

9. R. Kirchner, Nucl. Instr. Meth. B 70 (1992) 186

10. M. L. Huber, A. Laesecke, and D. G. Friend, NISTIR 6643 (2006) 